\title{
A Comparative Study of the Advance of the Newtonian Component of Mercury's Perihelion by Numerical Analysis and Perturbation Theory
}

\author{
Rajat Roy \\ Department of Electronics and Electrical Communication Engineering, Indian Institute of Technology, \\ Kharagpur, India \\ Email: rajatroy@ece.iitkgp.ernet.in
}

Received 1 July 2014; revised 5 August 2014; accepted 10 September 2014

Copyright (C) 2014 by author and OALib.

This work is licensed under the Creative Commons Attribution International License (CC BY).

http://creativecommons.org/licenses/by/4.0/

(c) (i) Open Access

\begin{abstract}
A new numerical evaluation of the Newtonian component of Mercury's perihelion advance over more than two centuries starting from about the year $\mathbf{2 0 0 0}$ is made. Results are given for about the last 30 years of this interval. Comparison with perturbation theory calculation results indicates necessity of revision of previously held estimates of this quantity.
\end{abstract}

Keywords

Celestial Mechanics, Numerical Solution of ODE, Mercury

Subject Areas: Mathematical Analysis, Quantum Mechanics

\section{Introduction}

Over the last few decades numerical computation of the dynamics of the planetary system and its application to the study of the orbital elements of Mercury have become commonplace. To give a few examples see [1] [2]. Numerical analysis can be more accurate than perturbation theory as it aims to solve the exact equation of motion with decreasing time step to enhance the accuracy whereas perturbation theory yields a fixed value of a quantity like the advance of Mercury's longitude of perihelion per century under the assumption that corrections to the two body problem are small. Also perturbation theory that has been used till today [3] cannot predict short term fluctuations in the advance as it is readily obtained in a numerical analysis (see Figure 1). We show that such a fluctuation in the Newtonian component of the perihelion advance when the total time period of study is slightly over two centuries from a suitable starting point can be quite large as the figure shows. This in our 


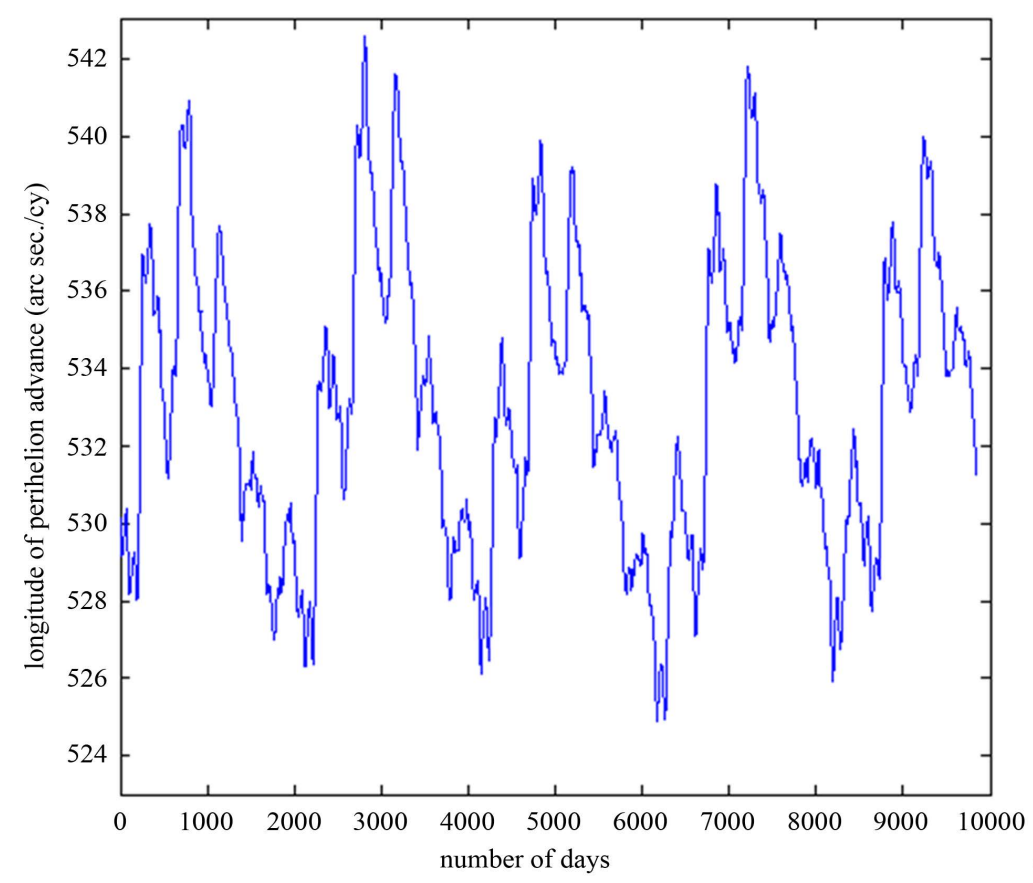

Figure 1. The advance in the longitude of perihelion of Mercury as obtained from a numerical solution of coupled system of $\mathrm{N}$ ordinary differential equations. The initial time is $J J=2451600.5$ and the total period of computation roughly 217 years. The plot is that for the last 10,000 days.

opinions can be source of error in the observation as the total time period of an accurate observation of this planet is hardly a few centuries old.

\section{Formulation of the Problem and Its Solution Using MATLAB Ordinary Differential Equation Solver}

The N-body problem of celestial mechanics can be formulated in terms of the heliocentric co-ordinate system and given for example by Arminjon [2] or Le Guyader [4] as

$$
\ddot{\vec{r}}=-G\left(m_{N}+m_{i}\right) \frac{\vec{r}_{i}}{R_{i}^{3}}+\sum_{\substack{j=1 \\ j \neq i}}^{N-1} G m_{j}\left(\frac{\overrightarrow{r_{j}}-\vec{r}_{i}}{\Delta_{i j}^{3}}-\frac{\overrightarrow{r_{j}}}{R_{j}^{3}}\right)(i=1 \cdots N-1)
$$

where all symbols have the same meaning as in reference [2]. The masses of the planets are taken from Rana [1] except that of Pluto which is a much smaller planet than what it was thought to be previously [4]. The initial or starting positions (and velocities) of all bodies on Julian ephemeris date $J J=2451600.5$ from reference [4] have been used. The heliocentric gravitational constant $G m_{N}$ is taken from the book by Taff [5]. The solver used is ODE113 of MATAB as recommended by Arminjon [2] and we accept RelTol $=5 \times 10^{-13}$ and $\mathrm{AbsTol}=10^{-15}$. Given the small mass of Pluto we have neglected it altogether and considered the eight body system excluding Sun. The post processing needed to calculate the longitude of perihelion of Mercury from the coordinates and velocity of the planet at any point of time is obtained by calculating first the elements of the osculating ellipse $a$ and $e$. From the direction of the angular momentum vector with respect to the ecliptic it is possible by the application of vector product law to locate the direction of the ascending node. The scalar product can then be used to find the argument of latitude and finally the true anomaly from the property of the (osculating) ellipse [5]. The longitude of perihelion is the quantity (longitude of ascending node + argument of latitude - true anomaly). Most perturbation calculations as we will see in the next section do not take this three dimensional nature of this quantity called the longitude of perihelion into consideration while applying it to Mercury and hence we need to apply corrections to the results quoted in these computations before comparing with our numerical calculations. 


\section{Results Obtained in Our Computations and Those by the Perturbation Method}

First we present the longitude of perihelion advance per century from the initial or the starting date of computations quoted in Section 2, to the final time which is about 217 years in the future from this starting date. Results are shown in Figure 1 for roughly a period of 27 years ending at the final date. In order that there is no confusion as to the quantity plotted along the y-axis in Figure 1, we state that it is the difference between the longitudes of perihelion (which has been defined in Section 2) at the time under consideration as shown in the figure and the longitude of perihelion at $J J=2451600.5$ is divided by the difference between the two times. Thus it is different from the quantity plotted in ref. [1] which has the unit arc sec. (and the one in the figure has the unit arc sec/cy). We find a fluctuation in the computed advance to be about 17 arc sec/cy from a mean value of 532.8 arc sec/cy. If we add this mean to the general relativistic contribution to the advance estimated by Stewart [3] to be $42.98 \mathrm{arc} \mathrm{sec/cy,} \mathrm{we} \mathrm{exceed} \mathrm{the} \mathrm{observed} \mathrm{value} \mathrm{of} \mathrm{the} \mathrm{precession} \mathrm{rate} \mathrm{slightly.} \mathrm{Moreover} \mathrm{what} \mathrm{is} \mathrm{to} \mathrm{be} \mathrm{kept} \mathrm{in}$ mind is that the observed advance (per century) will depend on exactly when the observation is made since the fluctuations in Figure 1 are quite rapid. These fluctuations will certainly reduce if we calculate the precession rate over a longer time period but time period of accurate observational astronomy is only a few centuries in the past.

From a study of this figure, the least claimed observed value of 575 arc sec/cy [3] for the longitude of perihelion advance of Mercury is within an error of \pm 3 arc sec/cy. Let us now turn to perturbation calculations based on Laplace-Runge-Lenz vector as presented in ref. [3]. The angular velocity of this quantity gives a value of its advance per unit time which is strictly confined to the plane of Mercury's orbit. Any change in the orientation of the orbital plane and its consequent effect on the node and through this on the longitude of perihelion is totally neglected. In fact the value obtained by Stewart [3] which is 532.23 arc sec/cy needs to be corrected for the motion of the node which yields an extra -3.35 arc sec/cy. The actual value of longitude of perihelion advance is only about 528.9 arc sec/cy from perturbation theory. To see how we obtain the quantity -3.35 arc sec/cy we write the full expression for the advance of Mercury's longitude of perihelion as given by Taff [5] for example (see page 314 of this reference)

$$
\frac{\mathrm{d} \varpi}{\mathrm{d} t}=\frac{\left(1-e^{2}\right)^{1 / 2}}{n a e}\left[-\mathcal{R} \cos v+\mathcal{T}\left(\frac{r}{p}+1\right) \sin v\right]+2 \frac{\mathrm{d} \Omega}{\mathrm{d} t} \sin ^{2}(i / 2)
$$

where all notations used are explained in this reference. Now the terms $\mathcal{R}$ and $\mathcal{T}$ are related to the radial and tangential (that is perpendicular to the radial) perturbing force in the orbital plane and hence must yield the same result as the motion of the Laplace-Runge-Lenz vector. The term $2 \frac{\mathrm{d} \Omega}{\mathrm{d} t} \sin ^{2}(i / 2)$ is on the other hand related to the motion of the node and the value of $\frac{\mathrm{d} \Omega}{\mathrm{d} t}$ as given by Rana [1] which is -451 arc sec/cy will yield -3.35 arc sec/cy for this extra term in Equation (2). In fact only the $\mathcal{R}$ and $\mathcal{T}$ contributions to precession that had been used in ref. [3] can be verified by studying Equation (4.2) of this reference and the sentences which appear below it. Finally in conclusion we take a look at a recent contribution in published literature by Smulsky [6]. This author states that the observed perihelion advance is not 575 arc sec/cy but somewhere around $582.5 \mathrm{arc} \mathrm{sec/cy.} \mathrm{Although} \mathrm{the} \mathrm{author} \mathrm{has} \mathrm{rightly} \mathrm{pointed} \mathrm{out} \mathrm{that} \mathrm{the} \mathrm{ecliptic} \mathrm{is} \mathrm{not} \mathrm{stationary} \mathrm{with} \mathrm{respect} \mathrm{to} \mathrm{a}$ fixed co-ordinate system that cannot be identified with saying the $\mathrm{x}-\mathrm{y}$ plane eternally, its motion is so small that it cannot make a difference in Mercury's perihelion precession rate by about 7 to 8 arc sec/cy. Right now our code is not equipped with the calculation of the inclination of the orbital plane of the earth but it can be easily incorporated in the near future to prove our point. We feel that other concerns like decreasing the time step of integration is more urgent for more accurate results and this has been pointed out by one of the referees.

\section{References}

[1] Rana, N.C. (1987) An Investigation of the Motions of the Node and Perihelion of Mercury. Astronomy \& Astrophysics, 181, 195-202.

[2] Arminjon, M. (2004) A Numerical Solution of the Inverse Problem in Classical Celestial Mechanics, with Application to Mercury’s Motion. Meccanica, 39, 17-29. http://dx.doi.org/10.1023/A:1026264517370

[3] Stewart, M.G. (2005) Precession of the Perihelion of Mercury’s Orbit. American Journal of Physics, 73, 730-734. 
http://dx.doi.org/10.1119/1.1949625

[4] Le Guyader, C. (1993) Solution of the N-Body Problem Expanded into Taylor Series of High Orders. Applications to the Solar System over Large Time Range. Astronomy \& Astrophysics, 272, 687-694.

[5] Taff, L.G. (1985) Celestial Mechanics: A Computational Guide for the Practitioner. John Wiley \& Sons, Inc., New York.

[6] Smulsky, J.J. (2011) New Components of the Mercury’s Perihelion Precession. Natural Science, 3, 268-274. http://dx.doi.org/10.4236/ns.2011.34034 